\title{
SERO-EPIDEMIOLOGY OF DENGUE VIRUS INFECTION IN CLINICALLY SUSPECTED PATIENTS ATTENDED IN DHAKA MEDICAL COLLEGE HOSPITAL DURING JANUARY TO DECEMBER 2016
}

\author{
PERVIN $M^{1}$, AKBAR AS ${ }^{2}$, HOSSAIN MZ ${ }^{3}$, SHARMIN ${ }^{4}$, FATEMA ${ }^{5}$, RAHMAN MA ${ }^{6}$, NEHAR N $^{7}$,
} YASMIN ${ }^{8}$, KHODA MME ${ }^{9}$, RAHMAN KM ${ }^{10}$, AZAD KAK ${ }^{11}$

\begin{abstract}
:
Introduction: Dengue is now established as one of the most important Arboviral infection in tropical countries. As the epidemic continues worldwide, this Aedes mosquito-transmitted pathogen is considered a major re-emerging tropical disease and significant public health concern. The study was conducted to determine the sero-epidemiology and clinical picture of dengue virus infection among the clinically suspected patients attended in Dhaka Medical College Hospital during January to December 2016.

Materials and Methods: The study was carried out on 145 clinically suspected Dengue patients attended in Dhaka Medical College Hospital from January to December 2016 who had fever with temperature $>39^{\circ} \mathrm{C}$. Blood sample was collected during acute febrile phase of patients and separated sera were tested for NS1 dengue antigen and IgM antibodies using commercial test kits (NS1 by OMC Healthcare (Pvt.) Ltd \& IgM antibody by Omega Diagnostics Ltd.).

Results: Of the total 145 patients 40 (27.6\%) were positive for Dengue NS1 antigen and or IgM antibody test. Of the positive 40 patients, 18 (45\%) were positive for NS1, 19 (47.5\%) were positive for IgM and 3 (7.5\%) were positive for both NS1 and IgM antibody. Major clinical manifestations were fever with arthralgia (79.5\%) and retro-orbital pain (50\%).

Conclusion: Outbreak of Dengue fever is continuing every year and typical presentation of Dengue fever has changed. Arthralgia and retro orbital pain was the most common clinical feature in the present study instead of headache and rash with fever. A large number of suspected dengue cases were negative by Dengue tests for antigen NS1 and IgM antibody which suggests other organisms of similar clinical manifestations are becoming prevalent in Dhaka city.
\end{abstract}

Key words: Dengue Fever, Dengue NS1 antigen, IgM antibody, Clinical feature of Dengue.

J Dhaka Med Coll. 2017; 26(2) : 111-116

\section{Introduction:}

Dengue is a common mosquito-borne arboviral disease of human worldwide. Almost 2.5 billion people are at risk of infection in the tropical and subtropical regions ${ }^{1}$. About 50 million infections occur globally every year ${ }^{2}$. The global prevalence of dengue has grown dramatically in recent years ${ }^{3}$ as the disease is spreading to new areas and its epidemiological pattern is gradually changing ${ }^{3,4,5}$.
The first Dengue cases were reported in Bangladesh in 1964 and the outbreak was known as 'Dacca Fever' ${ }^{6}$. A long period after that, dengue cases were undetected due to lack of diagnostic facilities. A few cases were reported in 1999 before a large outbreak occurred in 2000. During the outbreak 5,551 cases and 93 dengue-related deaths were reported ${ }^{7,8}$. From then onwards, Dengue outbreaks have occurred

1. Dr. Monira Pervin, Associate Professor and Head, Department of Virology, Dhaka Medical College.

2. Dr. Afroza Akbar Sweety, Assistant Professor, Department of Virology, Dhaka Medical College.

3. Dr. Mohammad Zaid Hossain, Associate Professor, Department of Medicine, Dhaka Medical College.

4. Dr. Rabeya Sharmin, Assistant Professor, Department of Virology, Dhaka Medical College.

5. Dr. Nusrat Fatema, Lecturer, Department of Virology, Dhaka Medical College.

6. Dr. Md. Anisur Rahman, Lecturer, Department of Virology, Dhaka Medical College.

7. Dr. Naznin Nehar, Lecturer, Department of Virology, Dhaka Medical College.

8. Dr. Marufa Yasmin, Lecturer, Department of Virology, Dhaka Medical College.

9. Dr. Mohammad Mehfuz-E-Khoda, Haemodialysis Unit, BIRDEM, Dhaka

10. Dr. Kazi Monisur Rahman, Assistant Professor, Gastroenterology, BSMMU, Dhaka

11. Prof. Khan Abul Kalam Azad, Professor, Department of Medicine, Dhaka Medical College, Dhaka

Correspondence : Dr. Monira Pervin, Associate Professor and Head, Department of Virology, Dhaka Medical College, Dhaka - 1000, Bangladesh. Mobile : 01711538741, E mail: monira66@yahoo.com 
in small or large scales every year in Bangladesh $9,10,11,12$.

Dengue syndrome comprises four clinical entities including undifferentiated fever (UF), Dengue fever (DF), Dengue hemorrhagic fever (DHF) and Dengue Shock Syndrome (DSS) ${ }^{3}$. There are four serotypes of Dengue viruses (Type I, II, III, IV) and different serotypes have capability to produce severity of different intensities ${ }^{3,13}$. Infection in human by one serotype produces life-long immunity against re-infection by the same serotype ${ }^{2,14}$. Primary infection with any of the serotypes is typically mild and self-limiting called Classical Dengue fever. Recovery from infection is generally complete and confers lifelong homo-typic immunity ${ }^{3,14}$. Classical DF presents as a fever of 5-7 days with headache, retro-orbital pain, backache, skin rashes, arthralgia and myalgia 13,15 . Secondary infection with other dengue serotypes may produce severe form of life threatening disease; Dengue Hemorrhagic fever (DHF) and Dengue shock syndrome (DSS) ${ }^{3}$. Primary infection may rarely produce DHF and DSS $^{16}$.

Detection of anti-dengue IgM indicates recent dengue infection in both primary and secondary cases which typically appear at day 5 of infection in primary infection. Anti-dengue IgM develops earlier than IgG in primary infection and is usually detectable by day 5 of illness and wanes after 1-2 months ${ }^{15,17}$. Non-structural protein 1 (NS1) is detectable in earlier days of fever usually 1-5 days of onset ${ }^{18}$. NS1 antigen detection is available for diagnosis of Dengue infection (DI) in early days of infection even before the appearance of IgM antibody ${ }^{19}$. Therefore, this test serves as an aid in the clinical laboratory diagnosis of early dengue infections prior to the presence of IgM or IgG antibodies in patients with clinical symptoms consistent with dengue infection. In a dengue patient, detection of only dengue specific IgM antibodies provides only partial information, denoting that the patient is suffering from acute dengue at least 5 days after the onset of fever. So the patient cannot be diagnosed at an early stage of the disease before that and also it cannot be classified whether he is suffering from primary or secondary dengue infection.
Therefore, in the present study both methods are used to diagnose acute dengue infection.

The objective of the present study was to find out positive patients of acute dengue infection among the suspected febrile patients presenting at Dhaka Medical College Hospital, Bangladesh to determine the prevalence of DF patients and to inform the present clinical symptoms of dengue infection among clinically suspected patients.

\section{Materials and Methods:}

The present study was a cross-sectional study during January to December 2016. Suspected dengue patients were enrolled in the study after taking written consent from participates (in case of children bellow 18 years written consent from legal guardian were taken). According to specific inclusion criteria, 145 clinically suspected patients with fever (presenting with body temperature above $100^{\circ} \mathrm{F}$ at the time of blood sample collection) plus two or more signs and symptoms from the following: headache, retroorbital pain, sub-conjuctival bleeding, myalgia, arthralgia, rash, lethargy, abdominal pain, anorexia, nausea, vomiting, loose motion, rash melena and or other bleeding manifestations, those fulfilling the case-definition of dengue fever (DF) were included in the study ${ }^{2,3}$. The exclusion criteria were defined cases of febrile illness with definite sources of infection and history of bleeding tendency since birth obtained from the medical records and patients presenting with fever of more than 15 days.

Clinical data were collected through interviewing the patients or their attendants, also obtained from the medical records and entered to the preformatted data sheet and after meticulous physical examination of the patients conducted by a doctor. After taking all aseptic precaution $5 \mathrm{ml}$ of venous blood sample was collected from each patient and transferred to a properly labeled sterile clot activated tube. Serum sample was collected in labeled eppendorf tubes within 2-3 hours of blood collection by centrifugation of the blood sample and after proper inspection of sample that there was no haemolysis. The serum samples were preserved in a $-20^{\circ} \mathrm{C}$ fridge until the testing for 
NS1 antigen by Immuno chromatographic test (ICT) and IgM dengue antibody by ELISA method according to the instruction of the manufacturer. Dengue NS1 Ag kit (OMC Healthcare (Pvt.) Ltd, Canada) was used for NS1 and anti-dengue IgM (Omega Diagnostics Ltd, UK) kit was used to perform tests in patient's samples.

According to our case definition, dengue positive cases diagnosed by NS1antigen and or anti-dengue IgM ELISA positive tests. Cases with both NS1 and anti-dengue IgM ELISA negative tests were considered to have other febrile illness. The details of NS1, IgM, dengue antibody test results including the day of performing the tests were documented for analysis of result.

\section{Results:}

Out of 145 clinically suspected dengue patients, $40(27.6 \%)$ were positive for NS1 and or positive IgM antibodies. Of the 40 dengue positive patients 18 patients were positive for NS1 dengue antigen, 19 patients were positive for IgM antibody and 3 patients were positive for both NS1 and IgM antibody shown in Table 1.

\section{Table-I}

Dengue positive cases by NS1 antigen and IgM antibody tests $(N=40)$

\begin{tabular}{cccc}
\hline Total & $\begin{array}{c}\text { No. of NS1 } \\
\text { Positive }\end{array}$ & $\begin{array}{c}\text { No. of IgM } \\
\text { antibody positive NS1 and IgM }\end{array}$ & No. of both \\
cases only & cases only & Positive cases \\
\hline $40(100 \%) 18(45 \%)$ & $19(47.5 \%)$ & $3(7.5 \%)$ \\
\hline
\end{tabular}

Of the 40 dengue positive patients 25 (62.5\%) were male and $15(37.5 \%)$ were female shown in Table2. The present study demonstrated the male predominance in the occurrence of dengue fever (25 male vs 15 female). Among males maximum 22(55\%) cases were occurred in the age group of 11-40 years whereas in females there is no specific age predominance and infection occurred among different age groups between 01-50 years.
Table-II

Age and Sex distribution among acute dengue positive

\begin{tabular}{lccc}
\hline Age & Male & Female & Total \\
\hline 0-10 year & $01(2.5 \%)$ & $01(2.5 \%)$ & $02(5 \%)$ \\
11-20 year & $06(15 \%)$ & $04(10 \%)$ & $10(25 \%)$ \\
21-30 year & $10(25 \%)$ & $04(10 \%)$ & $14(35 \%)$ \\
31-40 year & $06(15 \%)$ & $02(5 \%)$ & $08(20 \%)$ \\
41-50 year & $01(2.5 \%)$ & $04(10 \%)$ & $05(12.5 \%)$ \\
51-60 year & $00(00 \%)$ & $00(00 \%)$ & $00(00 \%)$ \\
>60 year & $01(2.5 \%)$ & $00(00 \%)$ & $01(2.5 \%)$ \\
\hline Total & $25(62.5 \%)$ & $15(37.5 \%)$ & $40(100 \%)$ \\
\hline
\end{tabular}

The distribution of clinical manifestations in dengue cases is given in Table 3 . All the dengue patients had body temperature $>100 æ \% \mathrm{~F}$. Common symptoms included arthralgia (73\%), retro-orbital pain (50\%), lethargy $(30 \%)$ and abdominal pain (30\%). Other symptoms were anorexia nausea vomiting, headache, loose motion, sub-conjunctival haemorrhage, myalgia, rash, Jaundice and melena which were distributed in lower frequencies.

Table-III

Clinical manifestations observed among Dengue positive Patients $(N=40)$

\begin{tabular}{lc}
\hline Clinical features & $\begin{array}{c}\text { No of patients } \\
\mathrm{N}(\%)\end{array}$ \\
\hline Temperature $(>38 æ \% \mathrm{C})$ & $40(100 \%)$ \\
Arthralgia & $35(87.5 \%)$ \\
Retro-orbital pain & $20(50 \%)$ \\
Lethargy & $12(30 \%)$ \\
Abdominal pain & $12(30 \%)$ \\
Anorexia, nausea and vomiting & $11(27.5 \%)$ \\
Headache & $10(25 \%)$ \\
Loose motion & $10(25 \%)$ \\
Sub- conjunctival bleeding & $8(20 \%)$ \\
Myalgia & $5(12.5 \%)$ \\
Rash & $5(12.5 \%)$ \\
Jaundice & $5(12.5 \%)$ \\
Melena / Per rectal bleeding & $3(7.5 \%)$ \\
\hline
\end{tabular}


In the present study, Dengue patients were presented mostly from August to December 2016 and Dengue positive cases were found few in monsoon and majority in the post monsoon season showed in Figure 1.

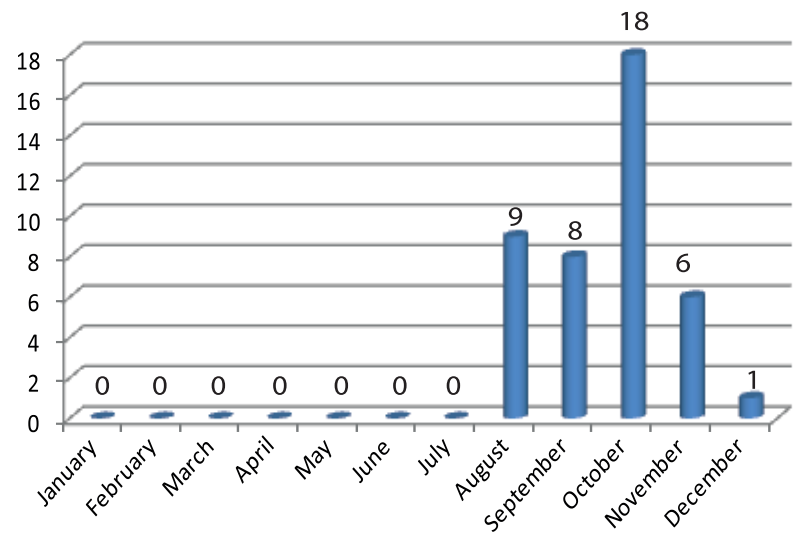

Fig.-1: Monthly distribution of Dengue positive patients $(N=40)$

\section{Discussion:}

Dengue is one of the most under-reported tropical diseases. The lack of laboratory resources and the nonspecific clinical presentation of non-severe cases greatly contribute to this situation. In the present study, out of 145 clinically suspected patients only $40(27.59 \%)$ patient's sera were positive for IgM antibody and or NS1 antigen. This low rate of positive result may indicate other viral infections having similar clinical presentation are prevailing at the same time. The massive outbreak of Chikungunya in 2017 is supporting the assumption of fever having similar clinical manifestations was present at the similar season and was spreading by a common mosquito vector Aedes aegeypti 20,21. A study carried out in a Medical College Hospital in Chittagong, Bangladesh in 2009-2010 showed low (39.96\%) positivity of Dengue IgM antibody among clinically suspected cases ${ }^{22}$. However, the rate of positive test for dengue was a little higher than the present study. In contrast, a very high $(92.4 \%)$ positivity of dengue antibody was found among the patients in Dhaka city in $2000^{9}$ indicating that the disease is now maintaining a low endemic state in Bangladesh.

The present study showed infection occurred mainly in young adult age. Maximum 14 (35\%) positive cases were in the age of 21-30 years. In another study from Bangladesh, Pervin et al showed the involvement of all age groups, especially an adult predominance with the mean age of the dengue patients was $29.2 \pm 12.9$ years and majority belonged to the 20-29 year of age 9 . Similar observation was found in many other studies in Bangladesh and surrounding countries ${ }^{5,23}$.

The present study is showing male predominance about $62.5 \%$ was male and $37.5 \%$ was female. Most of them (25\%) were in the age group of 21 to 30 years. Other studies from Singapore, India and Bangladesh showed similar male predominance in occurrence of infection which is also comparable to the present study $23,24,5$. However, this difference may not indicate more susceptibility of male to Dengue infection, because females in these areas are less privileged and may not get equal opportunity to have treatment for fever. In 2000 out break there was no significant difference in sex among dengue cases in Dhaka Bangladesh 9. In addition, reports from South America showed either equal proportions of male and female dengue cases or a greater proportion of female cases 25,26 . Other reasons of male predominance might be working adults who are symptomatic would seek medical attention from a hospital as they require medical certification to be excused from work. In addition, they are probably the only earning person to maintain family cost get more attention from other members of the family to seek medical care.

In the present study the common symptoms were arthralgia (73\%), retro-orbital pain $(50 \%)$, lethargy and abdominal pain (30\%) and anorexia, nausea, vomiting $(27.5 \%)$ in dengue infection cases. In an earlier study from Bangladesh showed myalgia (84.5\%), headache $(82.5 \%)$, arthralgia (68\%), lethargy (80.4\%) and retro-orbital pain $(49.5 \%)$ were the common symptoms ${ }^{9}$. A Study in India reported the common symptoms were headache $(92 \%)$ followed by myalgia (89\%), arthralgia (86\%) and retro orbital pain $(44 \%)$ in dengue cases ${ }^{24}$. A similar study in India also reported higher frequency of headache, myalgia and retroorbital pain in Dengue patients. However 
arthralgia was present in only $31 \%$ of patients in this study 27.

Headache and Rashes were important presenting symptoms in many studies $9,11,27$. However in the present study 25\% patients complained headache and rash was found only in few $(12.5 \%)$ cases. These results indicate the changes in the clinical presentation of DF patients in the current time.

The best environmental conditions for mosquitoes breeding prevail during pre and post monsoon periods in the tropical zones 28 . Although, in previous years the peak season of Dengue was found in the month of August 5,9,28, in the present study most of the DF patients presented in post monsoon during August to October 2016 and the peak incidence were found in October. This is possibly due to the change in the climate when the rainy season was short and intermittent rain occurs during the post monsoon in this rainy season.

There were some limitations in the present study that we could not exclude Chikungunya, other mosquito borne flavivirus and alphavirus infections and we could not also perform IgG antibody test to differentiate primary from secondary dengue infection due to our limited resource. A large scale study will have to be conducted in the future to describe the risk factors for the acquisition of dengue infection such as specific environmental, virological and human behavioral practice which could contribute in the changing epidemiology of dengue in Bangladesh.

\section{Conclusion:}

Outbreak of Dengue fever is continuing every year and typical presentation of DF has changed. Arthralgia and retro orbital pain is the most common clinical feature in the present dengue outbreak instead of headache and rash with fever. A large number of suspected dengue cases were negative by dengue tests for antigen NS1 and IgM antibody suggests other infections of similar clinical presentations are becoming prevalent in Bangladesh.

\section{References:}

1. WHO. World Health Organization Scientific Working Group Report on Dengue, 2007.
2. WHO. Dengue: Guidelines for diagnosis, treatment, prevention and control. New edition; 2009.

3. World Health Organization. Dengue Hemorrhagic Fever: Diagnosis, Treatment, Prevention and Control. Second edition. Geneva: World Health Organization, 1997: 1-84.

4. Sharmin S, Viennet E, Glass Kand and Harley D. The emergence of dengue in Bangladesh: epidemiology, challenges, and future disease risk. Trans R Soc Trop Med Hyg. 2015;109(10): 1-9.

5. Morales I, Salje H, Saha S, and Gurley ES. Seasonal Distribution and Climatic Correlates of Dengue Disease in Dhaka, Bangladesh. Am. J. Trop. Med. Hyg. 2016; 94(6), 359-1361.

6. Aziz MA, Gorham JR and Gregg MB. "Dacca Fever"An outbreak of dengue. Pakistan Journal of Medical Research. 1967; 6: 83-92.

7. Yunus EB, Bangali AM, Mahmood MAH, Rahman MM, Chowdhury AR and Talukder RK. Dengue outbreak 2000 in Bangladesh: From speculation to reality and exercise. Dengue Bulletin. 2001; 25: 1520.

8. Amin MMM, Hussain AMZ, Nahar K, Chowdhury IA, Murshed M and Chowdhury SA. Sero-diagnosis of dengue infections in four metropolitan cities of Bangladesh. Dengue Bulletin. 2000; 24: 34-41.

9. Pervin M, Tabassum S, Ali MM, Mamun KZ and Islam MN. Clinical and Laboratory Observations associated with the 2000 Dengue Outbreak in Dhaka, Bangladesh. Dengue Bulletin. 2004; 28: 96-106.

10. Sultana N, Biswas SK, Sultan T, Ahmed S,Hossain $Z$, Chowdhury R. Seroprevalence of dengue fever in Chittagong, Bangladesh. Chatt Maa Shi Hosp Med Coll J. 2013; 12(1): 38-40.

11. Sharmin R, Tabassum S, Mamun KZ, NessaA and Jahan M. Dengue infection in Dhaka City, Bangladesh. Mymensing Medical College Journal. 2013; 22(4):781-786.

12. Dhar-Chowdhury P, Paul KK, Haque CE, Hossain S, Lindsay LR, Dibernardo A, Brooks AW, Drebot MA. Dengue seroprevalence, seroconversion and risk factors in Dhaka, Bangladesh. PLoS Negl Trop Dis. 2017;11(3): e0005475. https://doi.org/10.1371/ journal.

13. Kuberski T, Rosen L, Reed D and Mataika J. Clinical and laboratory observations on patients with primary and secondary dengue type I infections with hemorrhagic manifestations in Fiji. The American Journal of Tropical Medicine and Hygiene. 1977; 26(4): 775-783.

14. Sabin AB. Research on Dengue during World War II. Am. J. Trop. Med. Hyg. 1952; 1: 30-50.

15. Innis BL, Nisalak A, Nimmannitya S,Kusalerdchariya S, Chongswasdi V,Suntayakorn S, Puttisri P and 
Hoke $\mathrm{CH}$. An enzyme-linked immunosorbent assay to characterize dengue infections where dengue and Japanese encephalitis co-circulate. The Am J. of Trop. Med. and Hyg, 1989; 40: 418-427.

16. Streatfield R, Bielby G, Sinclair D et al. A primary dengue 2 epidemic with spontaneous hemorrhagic manifestations. Lancet 1993; 342: 560-561.

17. Kuno G, Gomez I and Gubler DJ. An ELISA procedure for the diagnosis of dengue infections. Journal of Virological Methods. 1991; 33: 101-113.

18. Young PR, Hilditch PA, Bletchly C and Halloran W. An antigen capture enzyme linked immunosorbent assay reveals high levels of the dengue virus protein NS1 in the sera of infected patients. J Clin Microbiol. 2000; 38: 1053-57.

19. Datta S and Wattal C. Dengue NS1 antigen detection: A useful tool in early diagnosis of dengue virus infection. Indian J Med Microbiol. 2010; 28:107-10.

20. Khatun S, Chakraborty A, Rahman M, Banu NN, Rahman MM, Hasan SMM, Luby SP and Gurley ES. An outbreak of Chikungunya in rural Bangladesh 2011. POLS Negl Trop Dis 2015; 9: (7) e0003907. doi:10.1371/journal.pntd.0003907.

21. Kabir I, Dhimal M, Müller R, Banik S and Haque U. The 2017 Dhaka Chikungunya outbreak. The Lancet Infectious Disease. 2017; 17:1118.
22. Sultana N, Biswas SK, Sultan T, Ahmed S, Hossain $Z$ and Chowdhury R. Seroprevalence of dengue fever in Chittagong, Bangladesh. Chatt Maa Shi Hosp Med Coll J. 2013; 12(1): 38-40.

23. Ooi EE. Changing Pattern of Dengue Transmission in Singapore. Dengue Bulletin. 2001; 25:40-44.

24. Sreejith MG and George P. Study on the diagnostic efficacy of Clinico-Laboratory Parameters in serologically diagnosed cases of Dengue Fever. International Journal of Recent Trends in Science and Technology.2014;11(1): 12-16.

25. Neff JM, Marris L, Gonzales-Alcover, R. et al. Dengue fever in Puerto Rican community. Am. J. of Epidemiology. 1967; 86: 162-184.

26. Likofsky WH, Calisher $\mathrm{CH}$, Michelson AL et al. An Epidemiologic study of Dengue type 2 in Puerto Rico. Am. J. Epidemiol. 1969; 97: 264-275.

27. Babaliche P \& Doshi D. Catching Dengue Early: Clinical features and laboratory markers of Dengue virus infection. Journal of the Association of Physicians of India. 2015; 63: 38-41.

28. Ahmed TU, Rahman G, Bashar K et al. Seasonal prevalence of dengue vector mosquitoes in Dhaka city, Bangladesh. Bangladesh J Zool. 2007; 35: 20512 . 